\title{
The Integration of Process Planning and Shop Floor Scheduling in Small Batch Part Manufacturing
}

\author{
W. H. M. Zijm - Submitted by H. J. J. Kals (1) \\ University of Twente, Dept. of Mechanical Engineering, Enschede, Netherlands \\ Received on January 9, 1995
}

\begin{abstract}
In this paper we explore possibilities to cut manufacturing leadtimes and to improve delivery performance in a small batch part manufacturing shop by integrating process planning and shop floor scheduling. Using a set of initial process plans (one for each order in the shop), we exploit a resource decomposition procedure to determine schedules to determine schedules which minimize the maximum lateness, given these process plans. If the resulting schedule is still unsatisfactory, a critical path analysis is performed to select jobs as candidates for alternative process plans. In this way, an excellent due date performance can be achieved. with a minimum of process planning and scheduling effort.
\end{abstract}

Keywords

Scheduling, Process Planning, Critical Path Method.

\section{Introduction}

Manufacturing processes and, as a consequence, manufacturing planning and control, have become increasingly complex during the last few decades. On the one hand market requirements tend towards more diversity, a higher quality and a fast and accurate delivery performance. Shortening of leadtimes, not only in manufacturing but also in design and process planning, has been addressed as a major competitive edge, cf. Blackburn (1). The rapid advancements in information technology and technical automation, on the other hand, have definitely increased the potential flexibility on the shop floor. Unfortunately, this potential flexibility is seldom used effectively, due to the inadequacy of many existing planning and control systems.

To elaborate on the latter point, let us focus on one particular market segment where flexibility (i.e. the need to efficiently develop and manufacture a large variety of products with short, reliable leadtimes) has become crucial. A typical example is a small batch parts manufacturing shop, operating as a supplier to an OEM in a make-to-order environment. The use of CAD and CAPP systems often enable these shops to quickly implement design changes or to develop alternative process plans, thus increasing flexibility significantly, see e.g. Van Houten (2). The fact that capacity planning and in particular shop floor scheduling have become multiresource in nature, on the other hand severely complicates the manufacturing planning and control process, cf. Meester and Zijm (3) or Tiemersma (4). Complex product routings and uncertainty about processing times may frustrate the achievement of an excellent due date performance. Reduction of complexity, e.g. by implementing group technology or cellular manufacturing concepts, may offer important advantages, cf. Wiendahl and Scholtissek (5), but such solutions are only possible in relatively stable manufacturing environments. Small batch or one-of-a-kind manufacturing facilities are generally facing a too diverse demand pattern to allow for such solutions. The key is then the development of a flexible planning and control instrument, able to cope with the above mentioned complexities.

In this paper, we concentrate on the description of a planning and scheduling system for small batch parts manufacturing, developed in such a way that an easy integration with process planning alternatives is possible, to exploit the potential flexibility at the shop floor to the utmost possible. The system is robust in the sense that small disturbances, as they often occur on the shop floor, do not affect the capacity plans or schedules, whereas it is able to quickly evaluate alternatives in case of major disturbances. More important however, the system is able to guarantee the best possible due date performance of any given set of orders with a given set of process plans (to be called jobs in the sequel), to suggest jab candidates for alternative process plans in case of an unacceptable lateness and to evaluate these alternatives (all done automatically). The system essentially is based on a resource decomposition approach (to keep problems relatively simple) and exploits a critical path analysis of any resulting schedule to select candidates for alternative processing ways (developing alternative process plans in advance for each shop order is generally not viewed as a realistic solution).

The shop floor planning and scheduling system presented here has been developed at the Production and Operations Management Group of the University of Twente and has been tested at various companies in the Netherlands. The system has now been implemented in the software package Jobplanner. In this short review, we only present a rather global description of the system, for details the reader is referred to Meester and Zijm $(3,6)$ and the references mentioned there. In addition, we present the theoretical basis underlying the integration with process planning. The corresponding integration module is currently under development but some early tests have been performed and will be reported here.

The literature on integration of process and production planning so far seems to be rather limited. One of the early studies concerns the work of Chryssolouris and Chan (7) on the assignment of resources to the various production tasks, called the MADEMA approach. Tönshof et al. (8) generate non-linear process plans that include alternatives and discuss the integration with scheduling (see also Schmidt and Kreutzfeldt, 9). Lenderink (10) again requires a set of alternative process plans in advance and next uses an integer programming formulation to determine a capacity plan. Unfortunately, numerical difficulties prevent the solution of realistic problem sizes. See also (10) for some further references on integration of process and production planning.

\section{Shop floor scheduling: a decomposition approach}

Before describing the basics of the shop floor scheduling system in more detail, we present an example and introduce some terminology. Figure 1 shows a schematic view of the parts manufacturing shop of El-O-Matic in 


\section{Raw Materials}

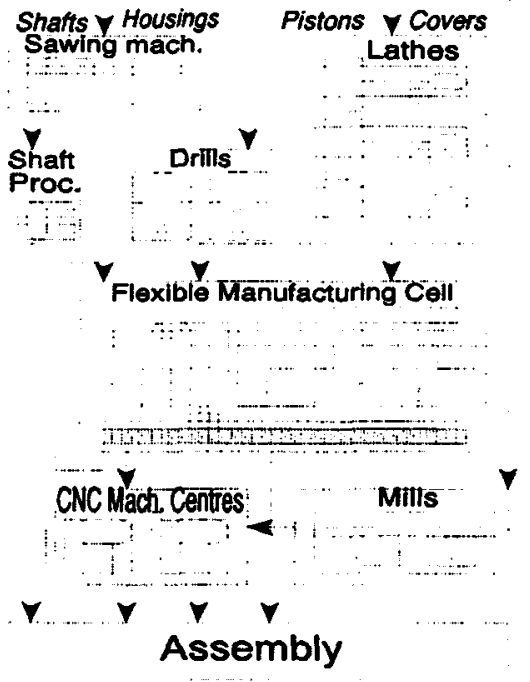

Figure 1. Parts manufacturing at EI-O-Matic

Hengelo, The Netherlands, an important supplier of electric and pneumatic actuators for valves (cf. Slomp and Zijm, 11). The shop consists of a number of functionally oriented machine groups, such as a drilling group, a group of NC lathes, a shaft processing step, a Flexible Manufacturing Cell (consisting of three CNC machining centres integrated with centralized tool store, tool robot, pallet pool and pallet transport device), some stand-alone CNC machining centres, etc. The main parts manufactured in the shop are the housings, the shafts, the pistons and the covers. The diversity of actuators (the variants) is to a large extent determined by the actuator housings (to be manufactured on the FMC) since these parts have to fit on the specific equipment of the customer. Besides machine capacity, the availability of unique cutting tools and pallet/fixture combinations severely limits the effective capacity of this FMC. The above described example serves as an illustration but the planning and scheduling approach is not limited to this example and will therefore be described in more general terms.

Next, we introduce some terminology. An order consists of a single product or a small batch of identical products which has to be manufactured. For each order, a high level process plan specifies a routing along a number of machining centres, possible auxiliary resource requirements (cutting tools, pallets, fixtures) at each centre and an estimate of the required processing time at each manufacturing stage. When a high level process plan is chosen, we speak of a job, whereas the processing at each manufacturing stage is termed an operation. Hence, a job generally represents a sequence of operations. A detailed process plan specifies all details of each operation at each centre (i.e. cutting patterns, speed and feed rates and the like) and forms the basis of an NC program. Hence, a high level process plan concerns the whole shop whereas a detailed process plan is associated with individual work centres. When discussing the integration of scheduling and process planning, the latter term refers exclusively to high level process planning

Now we turn to the basic decomposition mechanism underlying the planning and scheduling framework. Assume that a finite set of orders, with corresponding release and due dates (possibly different for each order) is given. Usually, these release and due dates follow from a higher level global planning system such as MRP (as in the case of El-O-Matic). For instance, the release dates reflect material availability dates, whereas the due dates are derived from the requested starting times of a subsequent assembly phase. Assume for the moment that for each order a process plan, specifying a routing, primary and auxiliary resource requirements and estimated processing times for each operation, is given (hence, in the terminology defined above we may now speak of jobs and operations). We wish to develop a schedule such that the maximum lateness (taken over all jobs) is minimal. The choice of minimizing maximum lateness, instead of average lateness, is motivated by industrial experiences. Minimizing average lateness may lead to some really late and other rather early jobs. generally viewed as a less satisfactory solution, in particular when various parts have to be combined in a subsequent assembly stage.

The decomposition procedure to be outlined below basically provides a mechanism to coordinate schedules of individual operations generated at an individual workcell. In our terminology, a workcell always represents a single manufacturing stage and may consist of a single machine, a set of parallel identical machines, one or more machines with additional set-up characteristics or even a Flexible Manufacturing Cell with additional tooling and fixturing constraints. In figure 1, each solid rectangle represents a workcell (yielding 7 workcells).

The basics of the coordination mechanism are best explained by using a small example with four jobs (with a total of ten operations) and three workcells (two cells consist of one machine each, one cell consists of two parallel identical machines, while no further resources are considered). Figure 3 gives the graphical representation of a partial schedule. The solid arcs represent precedence relations between operations of a job (induced by the routing), the dotted arcs represent precedence relations, following from partial schedules at workcells. Using longest path calculations in the graph, so-called virtual release and due dates for not yet scheduled operations in any workcell are now easily determined, which next may be used to determine a partial schedule for those operations. Once every workcell

A

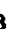

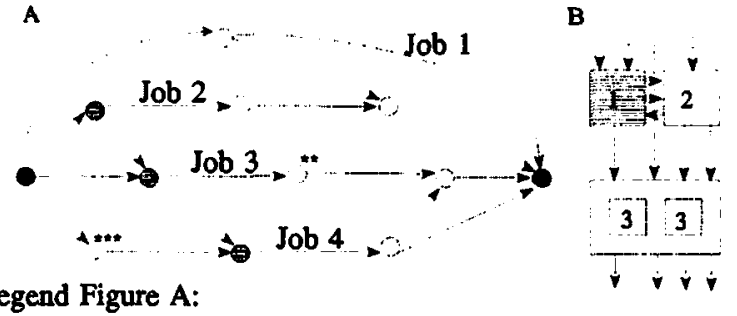

Legend Figure A:

$\bullet=$ Source $\bullet=$ W.C. 1 = W.C. 2 = W.C. $3 \quad=$ Sink

Legend Figure B:

$$
\triangle=\text { Job } 1,=\text { Job } 2,=\text { Job } 3 \ldots \text { = Job } 4
$$

Figure 2. A graph representation for a simple job shop schedule (W.C. = Workcell).

is scheduled, one may attempt to improve the overall schedule by relaxing the precedence relations between operations in any particular workcell, determine virtual release and due dates for the associated operations and next reschedule the cell. Continuing in this way, one may iterate between workcells until no further improvement is possible. Note that the critical path in the underlying graph automatically selects the operations which cause the largest lateness. This feature is the basis for the integration with process planning (cf. section 4). 
Summarizing, the partial schedules associated with each workcell naturally induce virtual release dates for succeeding operations on the routing of the jobs and virtual due dates for preceding operations. Note that such a coordination mechanism results in an integrated scheduling solution, in contrast to myopic priority rules, which only consider the capacity needs of each job separately, but which do not handle the interaction of these jobs at each workcell appropriately.

Up to now, we have explained how different single stage workcell schedules are coordinated. What has not been mentioned so far is how these individual workcells are scheduled such that the overall maximum lateness is minimized. The problem clearly is in the complexity of these single stage workcell scheduling problems and depends on the specific characteristics of the workcells: parallel machines, jobs with family-dependent setup times, a Flexible Manufacturing Cell with additional tool and fixture management problems, etc. Describing the techniques developed for various types of workcells is far beyond the scope of this paper, we refer to (6) and the references therein for a detailed overview as well as for a review of test results so far. The overall performance of the scheduling system appears to be excellent, in all cases investigated so far the results in terms of lateness of jobs were close to optimal. In addition, the system is rather quick; good solutions for problems with 200 jobs in a 50 machine shop (with on average 7 operations per job) were determined in less than 2 minutes on a PC with a 486 processor.

In the next section, we briefly describe how the decomposition mechanism can be extended to multiresource problems. In addition, a small summary of the most important workcell characteristics modelled so far, all motivated by industrial problems, will be presented.

\section{Multi-resource problems and further extensions}

The decomposition principle outlined in the preceding section applies equally well to multi-resource problems. To illustrate this, we focus on the Flexible Manufacturing Cell in the job shop of El-O-Matic (cf. figure 1, figure 3). Due to the presence of the tool robot, which is able to interchange tools between machine tool magazines and/or the central tool store, on-line (i.e. during machine processing), a drastic reduction of the number of duplicates of expensive cutting tools appeared to be possible. At El-O-Matic, more than $50 \%$ of the tools is unique. Obviously, job scheduling is now multi-resource in nature since both machine capacity and cutting tool availability have to be considered.

A detailed solution of this scheduling problem is described in Meester and Zijm (3). To handle the problem, they first

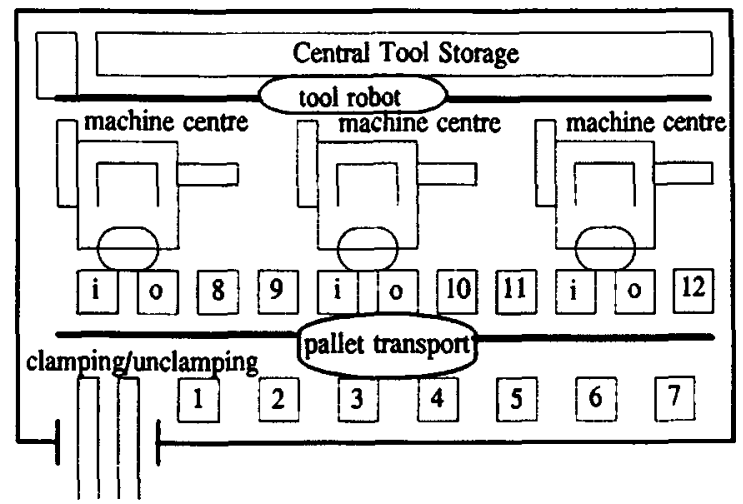

Figure 3. The Flexible Manufacturing Cell solve a so-called tool-exclusion problem, i.e. they ensure that no two jobs that need the same unique tool are scheduled simultaneously, by adjusting release and due dates accordingly. Next, based on the adapted (virtual) parameters, they solve a parallel machine scheduling problem, using a heuristic of Zijm and Nelissen (12). In passing we note that the need for sometimes dedicated pallet/fixture combinations adds some further resource constraints (3).

A classical multi-resource scheduling problem concerns the case of operators being responsible for multiple machines of various types (the so-called machineoperator interference problem). This situation was encountered at the machine shop of ERGON, a large electrotechnical installation company in Apeldoorn. The Netherlands. Basically, a decomposition technique similar to the one described in section 2 has been applied. Note that, in graph-theoretical terms, the presence of specially skilled operators induces additional arcs in each schedule, reflecting precedence relations between operations performed by each operator (possibly on different machines). Again, each (group of) operator(s) can be scheduled separately by adjusting virtual release and due dates of relevant jobs.

A further important extension concerns the inclusion of so-called family set-up times. In a situation in which setup means primarily machine set-up, it is natural to group jobs in families according to similar set-up characteristics. On the one hand, one wishes to cluster jobs belonging to the same family, in this way saving set-up times, on the other hand this may ruin the due date performance of jobs not belonging to this family (recall that jobs are subject to due dates and that we wish to minimize the maximum lateness). This trade-off between efficiency and due date performance has been observed to be a basic problem in many companies. An efficient branch and bound algorithm has been developed by Schutten et al. (13) and has been implemented in the general planning and scheduling framework. Implementations of the technique have been performed at the Sheet Metal Factory of DAF Trucks in Eindhoven, The Netherlands. Test results revealed a severe leadtime reduction, in particular in the Sheet Metal Press department.

Two case studies, performed at Morskate Actuators in Hengelo and at DAF Trucks in Eindhoven, stressed the need to jointly schedule the activities in the tool preparation room (calibration and assembly of tools with tool holders, adapters and the like) and the actual parts manufacturing shop. This extension is currently investigated.

Further extensions include the addition of transport delays, not only to cover actual transport times but merely to provide some robustness against small disturbances during execution of the jobs. Such delays are easily included in the decomposition procedure (basically the length of the arcs corresponding with the job routings are enlarged with the allowed delay whereas all other arcs remain unaltered). Indeed this inclusion of delays provides the desired robustness without delaying the job completion times much, as was revealed by studies performed at DAF Trucks and the machine shop of Stork Plastics Machinery in Hengelo, The Netherlands (producing a large variety of parts for injection moulding machines). One last extension concerns the possibility to adjust machine availability time windows, e.g. to include scheduled maintenance or the use of different shifts throughout the shop.

The procedures and algorithms described so far have been implemented in a complete multi-resource shop floor 
planning and scheduling system, called Jobplanner, which is now commercialized. The system basically consists of three layers, an automatic scheduler, an interactive scheduling mode and a monitoring and control system, while furthermore a central database, interfaces to the global planning system (often MRP) and the technical process planning system as weil as the shop floor (machine tools and further equipment) are provided. Figure 4 provides an overview of the system architecture.

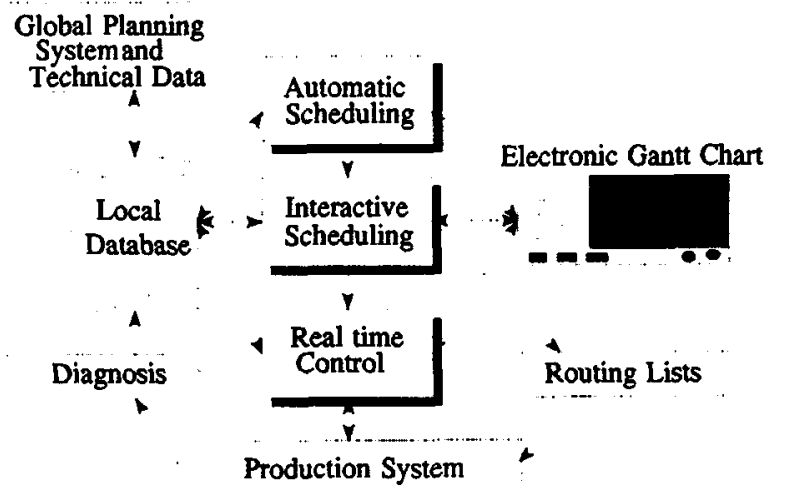

Figure 4. Architecture of JobPlanner

\section{Integration of scheduling and process planning}

In the introduction we already stressed the need to integrate process planning and shop floor scheduling, in particular for small batch parts manufacturing shops in which process planning is often performed only a few days before actual job execution. In classical process planning, job routings, required machines and cutting tools, and estimated processing times are often determined without taking into account the actual product mix on the shop floor during execution (although this product mix can be rather safely predicted). Using this knowledge might prevent unbalance and possible lateness of some jobs by considering the interaction of the process plans for different jobs.

The graph-theoretical representation, underlying the basic framework of Jobplanner, provides an almost ideal tool to select alternative process plans, based on a possible first realisation and evaluation of a production schedule. Recall that Jobplanner attempts to minimize the maximum lateness by calculating longest paths (critical paths) for each possible (partial) schedule. As a result, the system not only provides an optimal schedule, given a set of process plans, but also detects all operations which are on the critical path. Obviously, it is only necessary to consider alternatives for those operations; an alternative for any operation not on the critical path by definition yields no improvement. Finally, of all candidates, those alternative process plans are selected which yield a maximum decrease of the overall maximum lateness. In this way, we may iterate between scheduling and process planning until no further improvement occurs or the ultimate result is judged to be satisfactory.

The above procedure has been tested at the machine shop of Urenco in Almelo. The Netherlands, producing parts for ultra-centrifuges and environmental control systems for airplanes. Early test results revealed that in general only for a very small number of operations or jobresource combinations (typically less than one percent) alternative process plans have to be developed. The experiments at Urenco yielded an improvement of manufacturing leadtimes of 10 to $30 \%$. (cf. Wilbers, 14). Further experiments are currently under preparation.

\section{Conclusions}

In this paper, we have described the development of a planning and scheduling framework which provides close to optimal results for a set of jobs. The methodology allows for an easy integration with high level process planning. In case of an unsatisfactory due date performance, job candidates for alternative process plans are suggested and those alternatives are chosen which yield the best possible improvement in delivery performance.

\section{References}

1. Blackburn, J.D. [1991], Time-based competition: the next battleground in American Manufacturing. Irwin,

2. Van Houten, F.J.A.M [1991], PART, a computer aided process planning system, Ph.D Thesis, University of Twente, Department of Mechanical Engineering,

3. Meester, G.J. and W.H.M. Zijm [1993], Multi-resource scheduling of an FMC in discrete parts manufacturing, in $M$. Munir Ahmad and William G. Sullivan (eds.): Flexible Automation and Integrated manufacturing 1993, CRC Press Inc., Atlanta, pp. 360-370.

4. Tiemersma, J.J. [1992], Shop Floor Control in small batch part manufacturing. Ph.D Thesis, University of Twente, Department of Mechanical Engineering,

5. Wiendahi, H.-P. and P. Scholtissek [1994], Management and control of complexity in manufacturing, Annals of the CIRP, Vol. 43/2, pp. 1-8

6. Meester, G.J. and W.H.M. Zijm [1994], Development of a shop floor planning and scheduling system for hybrid job shops, Working Paper LPOM-94-1, Department of Mechanical Engineering, University of Twente, submitted to International Journal of Production Economics,

7. Chryssolouris, G. and S. Chan [1985], An integrated approach to process planning and scheduling. Annals of the CIRP, Vol. 34/1, pp. 413-415.

8. Tönshof, H.K., U. Beckendorff and N. Anders [1989], FLEXPLAN, a concept for intelligent process planning and scheduling, CIRP International Workshop on CAPP, Hannover University, September 21-22, 1989.

9. Schmidt, B.C. and J. Kreutzfeldt [1992], ESPRITProject 2457 FLEXPLAN: Integrated process planning and workshop scheduling, in: G.J. Olling and $F$. Kimura (eds.), Human Aspects in Computer Integrated Manufacturing, IFIP,

10. Lenderink, A. [1994], The integration of process planning and machine loading in small batch part manufacturing, Ph.D Thesis, University of Twente, Department of Mechanical Engineering,

11. Slomp, J. and W.H.M. Zijm [1993], A manufacturing planning and control system for a flexible manufacturing system, Robotics and Computer integrated Manufacturing 10, pp. 109-114.

12. Zijm, W.H.M. and E.H.L.B. Nelissen [1990], Scheduling a Flexible Machining Centre, Engineering Costs and Production Economics 19, pp. 249-258,

13. Schutten, J.M., S.L. van de Velde and W.H.M. Zijm [1993], Scheduling a single machine with release and due dates and family-dependent set-up times, Working Paper LPOM-93-4, Department of Mechanical Engineering, University of Twente, to 8appear in Management Science,

14. Wilbers, A.A.B. [1994], Capaciteitsplanning by Urenco Nederland B.V., Master Thesis, Laboratory of Production and Operations Management, Department of Mechanical Engineering, University of Twente (in Dutch). 